\title{
Fatores que Influenciam o Aluno na Escolha da Especialidade Médica
}

\author{
Factors that Influence the Student's Choice of \\ Medical Specialty
}

\author{
Paulo Roberto Corsi ${ }^{1}$ \\ Érika Lopes Fernandes ${ }^{I}$ \\ Priscila Marques Intelizano ${ }^{I}$ \\ Carla Carolina Borges Montagnini ${ }^{I}$ \\ Felipe Iankelevich Baracat ${ }^{I}$ \\ Manoel Carlos Sampaio de Almeida Ribeiro
}

\section{PALAVRAS-CHAVE}

- Medicina;

- Escolha da Profissão;

- Educação Médica;

- Estudantes.

\section{KEYWORDS}

- Medicine;

- Career Choice;

- Medical Education;

- Students.
Reencaminhado em: 22/07/2013

Reencaminhado em: 29/11/2013

Aprovado em: 19/01/2014

\section{RESUMO}

A escolha da especialidade define a carreira profissional de um médico. Pesquisas que visem analisar os fatores que impulsionam os alunos nessa decisão são importantes para entendermos os anseios dos estudantes e planejar estratégias educacionais correspondentes à necessidade do sistema de saúde brasileiro. O presente estudo analisou os fatores que influenciam a escolha da especialidade, correlacionando-os ao ano letivo e com aspectos socioeconômicos dos estudantes de Medicina da Faculdade de Ciências Médicas da Santa Casa de São Paulo (FCMSCSP). Trata-se de um estudo transversal, realizado com 456 alunos do primeiro ao sexto ano da FCMSCSP que responderam a um questionário, divididos em três grupos: primeiro ciclo (primeiro e segundo anos), segundo ciclo (terceiro e quarto anos) e terceiro ciclo (quinto e sexto anos). Os fatores estatisticamente significantes na comparação entre os ciclos foram: horas de trabalho, qualidade de vida, tempo livre para lazer, enriquecimento precoce, recompensa financeira, relação médico-paciente, conteúdo cognitivo da especialidade, conselhos de amigos e de parentes. Qualidade de vida, retorno financeiro e influências de terceiros foram os mais importantes para a escolha das especialidades.

\begin{abstract}
The choice of specialty defines the career of a doctor. Studies to analyze the factors that contribute to the student's decision are important to reveal the students' expectations and to plan strategies relevant to the needs of the Brazilian health system. The present study analyzed the factors that influence the choice of specialty correlating them to the academic year and socioeconomic aspects of medical students of the Faculdade de Ciências Médicas da Santa Casa de São Paulo (FCMSCSP). This is a cross-sectional study with 456 first to sixth year students at FCMSCSP who answered a questionnaire and were divided into 3 groups: First cycle (first and second years), second cycle (third and fourth years) and third (fifth and sixth years). Statistically significant factors in comparisons between the cycles were: hours of work, quality of life, free time for leisure activities, early enrichment, financial reward, doctor-patient relationship, the cognitive content of the specialty, advice from friends and relatives. Quality of life, financial return and third-party influences were shown to be the most important factors in the choice of specialties.
\end{abstract}




\section{INTRODUÇÃO}

O exercício profissional do médico é heterogêneo devido ao grande número de especialidades e subespecialidades existentes, que se caracterizam por diferenças na área de atuação, no ambiente de trabalho e na variedade dos pacientes ${ }^{1-4}$.

O Conselho Federal de Medicina, em 2011, reconheceu 53 especialidades médicas, que devem contar com no mínimo dois anos de formação, e 52 áreas de atuação, com no mínimo um ano de formação 5 .

A escolha da especialidade é uma importante decisão que define a carreira profissional de um médico. Um aluno de Medicina geralmente faz reflexões a respeito de sua personalidade, estilo de vida, valores pessoais e diversos interesses no caminho de tomar essa decisão $0^{6-12}$.

Diversos estudos têm sido realizados com a proposta de analisar os fatores que influenciam esta escolha. Quanto à personalidade, estudos anteriores mostraram que perfis psicológicos identificados na admissão ou durante o curso de Medicina podem ser preditores ou estar associados à especialidade escolhida pelo aluno ${ }^{13-25}$.

A partir da década de 1990, muitos estudos têm levado em consideração fatores como estilo de vida, recompensa financeira, desejo de prestígio social e efeito de mentores ${ }^{4,22}$. Muitos desses fatores podem ter modificado este cenário suficientemente para tornar as características psicológicas um fator menos importante na escolha de uma especialidade ${ }^{4}$. Fatores relacionados ao estilo de vida, tais como horas de trabalho, tempo livre para atividades e frequência de plantões noturnos, têm sido apontados como importantes na escolha da especialidade ${ }^{10,23,24}$.

Esse tema tem sido amplamente abordado, principalmente por faculdades norte-americanas e europeias, uma vez que as características e a distribuição da mão de obra médica nas especialidades afetam a manutenção e a evolução de qualquer sistema de saúde ${ }^{25}$.

No Brasil, existem poucos estudos sobre esse tema. Um estudo com alunos do último ano de oito escolas médicas do Estado de São Paulo verificou que quase a metade dos estudantes, ao ingressar na faculdade, já havia pensado sobre a especialidade. Para um quarto destes, a primeira escolha prevaleceu. Psiquiatria e Cirurgia foram as escolhas com maior porcentagem de estabilidade. Homens valorizaram o dinheiro, resultados terapêuticos imediatos e ter um emprego em instituições particulares. Mulheres atribuíram maior importância a uma carreira acadêmica e ter uma agenda mais regular. Quase a metade admitiu ter tido dificuldade na escolha da especialidade $^{26}$.
Outro estudo encontrou que no Brasil ainda há uma significativa diferença entre homens e mulheres na escolha das especialidades. Mulheres tendem para Pediatria, e homens, para Cirurgia e Ortopedia. Houve declínio de 1979 a 1984 na escolha de Clínica Médica em ambos os sexos e aumento na escolha de Anestesiologia entre homens e Radiologia entre mulheres ${ }^{27}$.

Um estudo que comparou razões de escolha da especialidade entre residentes de Clínica Médica e Cirurgia mostrou que as principais razões da Clínica foram contato com o paciente, gosto por atividades intelectuais e a abrangência da área. Já entre a Cirurgia, as principais razões de escolha foram o tipo de intervenção prática e objetiva, o gosto por atividades manuais e os resultados rápidos e concretos que a área proporciona. Características de personalidade influenciaram ambas as áreas, sem diferença estatística. $\mathrm{O}$ momento da escolha difere nas duas áreas, sendo que cirurgiões decidem optar pela especialidade mais precocemente, antes mesmo de ingressar na faculdade, enquanto clínicos decidem no internato (quinto e sexto anos) ${ }^{28}$. Em geral, os resultados obtidos no Brasil reafirmam estudos realizados em outros países com características sociais e educacionais bastante diversas ${ }^{28-38}$.

Visto o pequeno número de estudos brasileiros a respeito da escolha das especialidades médicas, tornam-se necessárias pesquisas nessa área para que possamos entender os anseios dos estudantes acerca da especialidade e planejar estratégias educacionais correspondentes à necessidade do sistema de saúde brasileiro.

\section{OBJETIVO}

O presente estudo pretende investigar os fatores que influenciam a escolha da especialidade, correlacionando-os ao ano letivo e com aspectos socioeconômicos dos estudantes de Medicina da Faculdade de Ciências Médicas da Santa Casa de São Paulo.

\section{MATERIAL E MÉTODO}

Trata-se de um estudo transversal, realizado com os alunos do primeiro ao sexto ano da Faculdade de Ciências Médicas da Santa Casa de São Paulo. Responderam ao questionário proposto 456 alunos durante o ano de 2011, após a assinatura do termo de consentimento livre e esclarecido.

\section{Instrumento}

Os alunos responderam voluntariamente a um questionário com questões objetivas sobre dados socioeconômicos, uma 
questão aberta para ser informada a especialidade médica pretendida e uma lista com diversos fatores, cujos impactos o aluno indicou na escolha da especialidade, variando de 1 a 5, sendo 1 "nada importante" e 5 "muito importante". Os fatores pesquisados fazem parte das esferas da qualidade de vida, retorno financeiro e profissional proporcionado pela especialidade, aspectos do dia a dia da carreira, relação médico-paciente e efeito de outras pessoas, como professores, amigos, médicos e parentes.

\section{Análise estatística}

As variáveis foram analisadas por meio dos testes do qui-quadrado e de Kruskal Wallis, utilizando um nível de significância de 0,05. Foram calculados intervalos de confiança de 95\% para as estimativas produzidas. Utilizou-se o programa SPSSv17 para o processamento e análise dos dados.

\section{RESULTADOS}

A amostra da pesquisa é composta por 91 (20\%) alunos do primeiro ano, 98 (21,5\%) do segundo ano, 68 (14,9\%) do terceiro ano, 90 (19,7\%) do quarto ano, 56 (12,3\%) do quinto ano e 53 $(11,6 \%)$ alunos do sexto ano. A porcentagem de alunos do sexo feminino foi $46,5 \%$, e do sexo masculino, $53,5 \%$. Os alunos foram divididos em três grupos: primeiro ciclo (primeiro e segundo anos), segundo ciclo (terceiro e quarto anos) e terceiro ciclo (quinto e sexto anos). O primeiro ciclo correspondeu a $41,4 \%$ dos alunos, o segundo ciclo a $34,6 \%$ e o terceiro ciclo a $23,9 \%$ dos alunos. A faixa etária da amostra variou de 18 a 43 anos, sendo $62,3 \%$ entre 21 e 24 anos.

A renda familiar mensal foi informada por $91 \%$ dos alunos e, dentre estes, foi visto que: $0,5 \%$ possuem renda menor que 1 mil reais; $16,6 \%$ renda entre 1 mil e 5 mil reais; $19,8 \%$ renda entre 5 mil e 10 mil reais; $17,1 \%$ renda entre 10 mil e 15 mil reais; $18,6 \%$ renda entre 15 mil e 20 mil reais; $15,7 \%$ renda entre 20 mil e 30 mil reais; e 11,8\% renda maior que 30 mil reais. Quanto a bolsa de estudos, 24,3\% dos alunos declararam possuí-la. A renda familiar foi analisada separadamente, de acordo com os seis grupos citados, para avaliar a influência na escolha da especialidade. Não houve diferença na escolha segundo o fator renda.

Os alunos cujo pai é médico correspondem a 30,5\% e cuja mãe é médica, a 13,4\%. A presença de algum parente médico (exceto pai, mãe e irmãos) foi observada em 43,6\% dos alunos.

Relataram experiência extrafaculdade, como instrumentador em cirurgias, 164 (36\%) alunos entrevistados.

Em relação à escolha da especialidade, 37,1\% dos alunos ainda não haviam escolhido a especialidade, 43,6\% escolhe- ram especialidades gerais (como Cirurgia Geral, Pediatria, Clinica Médica e Ginecologia e Obstetrícia) e 19,3\% especialidades específicas (Tabela 1).

TABELA 1

Características dos alunos $(\mathrm{n}=456)$, FCMSCSP, 2011

\begin{tabular}{|c|c|c|}
\hline Sexo & & \\
\hline Masculino & 244 & $53,5 \%$ \\
\hline Feminino & 212 & $46,5 \%$ \\
\hline \multicolumn{3}{|l|}{ Faixa etária } \\
\hline $18-20$ & 81 & $17,8 \%$ \\
\hline $21-24$ & 288 & $63,2 \%$ \\
\hline $25-43$ & 87 & $19,1 \%$ \\
\hline \multicolumn{3}{|l|}{ Ciclo } \\
\hline $1^{\mathrm{o}}$ & 189 & $41,4 \%$ \\
\hline $2^{\underline{0}}$ & 158 & $34,6 \%$ \\
\hline $3^{\circ}$ & 109 & $23,9 \%$ \\
\hline \multicolumn{3}{|l|}{ Possui bolsa $(n=454)$} \\
\hline Não & 343 & $75,2 \%$ \\
\hline Sim & 111 & $24,3 \%$ \\
\hline \multicolumn{3}{|l|}{ Renda familiar $(n=434)$} \\
\hline Até 5 mil & 90 & $19,7 \%$ \\
\hline 6 a 10 mil & 82 & $18,0 \%$ \\
\hline 10 a 15 mil & 70 & $15,4 \%$ \\
\hline 15 a 20 mil & 78 & $17,1 \%$ \\
\hline Mais de $20 \mathrm{mil}$ & 114 & $25,0 \%$ \\
\hline \multicolumn{3}{|l|}{ Pai ou mãe médicos } \\
\hline Não & 300 & $65,8 \%$ \\
\hline Sim & 156 & $34,2 \%$ \\
\hline \multicolumn{3}{|l|}{ Escolha da especialidade } \\
\hline Não escolheu & 169 & $37,1 \%$ \\
\hline Especialidades Gerais & 199 & $43,6 \%$ \\
\hline Especialidades Específicas & 88 & $19,3 \%$ \\
\hline
\end{tabular}

Os fatores relacionados à escolha da especialidade foram avaliados pelos alunos quanto a sua importância na influência e correlacionados com o ciclo a que o aluno pertence. Foram 
positivamente avaliados quanto a sua influência na escolha da especialidade e estatisticamente significantes na comparação entre os ciclos, sendo mais valorizados: conselhos de amigos, lazer, interação com outros médicos, conselhos de parentes, estilo de vida durante a residência, admiração a um mentor, departamento renomado e foco em saúde pública. Destes, os mais importantes para o primeiro ciclo são estilo de vida e foco em saúde pública. Os demais são mais relevantes para o terceiro ciclo (Tabela 2).

\section{TABELA 2}

Medianas dos escores de importância dos fatores relacionados à escolha da especialidade médica segundo o ciclo, FCMSCSP, 2011.

\begin{tabular}{|c|c|c|c|c|}
\hline Fatores & $\begin{array}{c}11^{\circ} \\
\text { Ciclo }\end{array}$ & $\begin{array}{l}2^{2} \\
\text { Ciclo }\end{array}$ & $\begin{array}{c}3^{\circ} \\
\text { Ciclo }\end{array}$ & $\mathrm{p}$ \\
\hline Conselhos de amigos & 2 & 2 & 3 & 0,001 \\
\hline Tempo para lazer & 4 & 3 & 4 & 0,007 \\
\hline Interação com outros médicos & 3 & 3 & 3 & 0,013 \\
\hline Conselhos de parentes & 2 & 2 & 3 & 0,023 \\
\hline Estilo de vida durante a residência & 3 & 2 & 3 & 0,024 \\
\hline Admiração a um mentor & 2 & 2 & 3 & 0,031 \\
\hline Departamento renomado & 2 & 2 & 3 & 0,035 \\
\hline Foco em saúde pública & 2 & 2 & 2 & 0,04 \\
\hline Horas de trabalho & 3 & 3 & 4 & 0,054 \\
\hline Recompensa financeira & 4 & 4 & 4 & 0,062 \\
\hline $\begin{array}{l}\text { Distribuição por sexo da } \\
\text { especialidade }\end{array}$ & 1 & 1 & 1 & 0,071 \\
\hline Interesse em pesquisa & 2 & 2 & 2 & 0,085 \\
\hline Duração da residência & 2 & 2 & 2 & 0,089 \\
\hline Conselhos de outros médicos & 3 & 3 & 3 & 0,098 \\
\hline $\begin{array}{l}\text { Compreender paciente como um } \\
\text { todo }\end{array}$ & 4 & 4 & 3 & 0,115 \\
\hline $\begin{array}{l}\text { Conteúdo intelectual da } \\
\text { especialidade }\end{array}$ & 4 & 4 & 4 & 0,137 \\
\hline Flexibilidade da especialidade & 3 & 3 & 4 & 0,221 \\
\hline Conselhos de professores & 2 & 2 & 3 & 0,312 \\
\hline
\end{tabular}

Segundo o critério possuir bolsa ou não de estudo, os fatores mais relevantes, em ordem decrescente, na escolha da especialidade foram: faturamento precoce, estilo de vida duran- te a residência, duração da residência, foco em saúde pública e qualidade de vida. Dentre esses, os mais relevantes para os não bolsistas foram: faturamento precoce, estilo de vida durante a residência, foco em saúde pública e qualidade de vida. Para os bolsistas, o mais relevante foi a duração da residência (Tabela 3).

TABEla 3

Medianas dos escores de importância dos fatores relacionados à escolha da especialidade médica segundo bolsa, FCMSCSP, 2011.

\begin{tabular}{|c|c|c|c|}
\hline Fatores & Não-bolsista & Bolsista & $\mathrm{p}$ \\
\hline Faturamento precoce & 3 & 3 & 0,021 \\
\hline $\begin{array}{l}\text { Estilo de vida durante a } \\
\text { residência }\end{array}$ & 3 & 3 & 0,026 \\
\hline Duração da residência & 2 & 3 & 0,03 \\
\hline Foco em saúde pública & 2 & 2 & 0,038 \\
\hline Qualidade de vida & 5 & 4 & 0,04 \\
\hline Foco em emergência & 3 & 3 & 0,057 \\
\hline Interação com outros médicos & 3 & 3 & 0,059 \\
\hline Conselhos de amigos & 2 & 2 & 0,115 \\
\hline Flexibilidade da especialidade & 3 & 4 & 0,123 \\
\hline Tempo para lazer & 4 & 4 & 0,138 \\
\hline Conselhos de professores & 2 & 3 & 0,164 \\
\hline Recompensa financeira & 4 & 4 & 0,179 \\
\hline Áreas de atuação & 4 & 4 & 0,24 \\
\hline Conselhos de parentes & 2 & 2 & 0,257 \\
\hline Diversidade de pacientes & 3 & 3 & 0,319 \\
\hline A pressão do dia a dia & 3 & 3 & 0,329 \\
\hline Horas de trabalho & 3 & 3 & 0,357 \\
\hline Prestígio da especialidade & 3 & 3 & 0,389 \\
\hline
\end{tabular}

Quanto ao sexo, os fatores mais relevantes para os homens foram: foco em emergência, oportunidade de construir carreira, recompensa financeira, conteúdo intelectual da especialidade, departamento renomado, prestígio e diversidade 
de pacientes. Já para as mulheres foram: horas de trabalho, qualidade de vida, tempo para se dedicar à família, pressão do dia a dia, distribuição por sexo da especialidade, compreender o paciente como um todo (biopsicossocial), foco em saúde pública, relação médico-paciente, estilo de vida durante a residência (Tabela 4 ).

TABELA 4

Medianas dos escores de importância dos fatores relacionados à escolha da especialidade médica segundo sexo, FCMSCSP, 2011.

\begin{tabular}{|c|c|c|c|}
\hline Fatores & Feminino & Masculino & $\mathbf{p}$ \\
\hline Horas de trabalho & 4 & 3 & 0,000 \\
\hline Qualidade de vida & 5 & 4 & 0,000 \\
\hline Tempo para se dedicar à família & 5 & 4 & 0,000 \\
\hline A pressão do dia a dia & 3 & 3 & 0,000 \\
\hline $\begin{array}{l}\text { Distribuição por sexo da } \\
\text { especialidade }\end{array}$ & 1 & 1 & 0,000 \\
\hline $\begin{array}{l}\text { Compreender paciente como } \\
\text { um todo }\end{array}$ & 4 & 3 & 0,000 \\
\hline $\begin{array}{l}\text { Relação a longo prazo com o } \\
\text { paciente }\end{array}$ & 3 & 3 & 0,000 \\
\hline Foco em saúde pública & 2 & 2 & 0,000 \\
\hline Foco em emergência & 2 & 3 & 0,000 \\
\hline Oportunidade de emprego & 4 & 4 & 0,001 \\
\hline Recompensa financeira & 4 & 4 & 0,001 \\
\hline Relação médico-paciente & 4 & 4 & 0,001 \\
\hline $\begin{array}{l}\text { Conteúdo intelectual da } \\
\text { especialidade }\end{array}$ & 4 & 4 & 0,002 \\
\hline Departamento renomado & 2 & 3 & 0,008 \\
\hline $\begin{array}{l}\text { Estilo de vida durante a } \\
\text { residência }\end{array}$ & 3 & 2 & 0,010 \\
\hline Prestígio da especialidade & 3 & 3 & 0,015 \\
\hline Diversidade de pacientes & 3 & 3 & 0,026 \\
\hline $\begin{array}{l}\text { Dificuldade para entrar na } \\
\text { residência }\end{array}$ & 2 & 2 & 0,060 \\
\hline
\end{tabular}

No fator renda, os critérios mais relevantes foram dificuldade para entrar na residência, estilo de vida durante a residência e faturamento precoce, sendo o último mais relevante quanto menor a renda (Tabela 5).
TABELA 5

Medianas dos escores de importância dos fatores relacionados à escolha da especialidade médica segundo renda, FCMSCSP, 2011.

\begin{tabular}{|c|c|c|c|c|c|c|}
\hline Fatores & $\begin{array}{c}\text { Até } 5 \\
\text { mil }\end{array}$ & $\begin{array}{c}6 \text { a } 10 \\
\text { mil }\end{array}$ & $\begin{array}{c}10 \text { a } \\
15 \\
\text { mil }\end{array}$ & $\begin{array}{c}15 \text { a } \\
20 \\
\text { mil }\end{array}$ & $\begin{array}{c}\text { Mais } \\
\text { de } 20 \\
\text { mil }\end{array}$ & $\mathrm{p}$ \\
\hline $\begin{array}{l}\text { Dificuldade para } \\
\text { entrar na residência }\end{array}$ & 2 & 2 & 3 & 2 & 2 & 0,009 \\
\hline $\begin{array}{l}\text { Estilo de vida } \\
\text { durante a residência }\end{array}$ & 3 & 3 & 3 & 3 & 2 & 0,025 \\
\hline Faturamento precoce & 3 & 3 & 2 & 3 & 2 & 0,048 \\
\hline Tempo para lazer & 3 & 4 & 3 & 4 & 4 & 0,067 \\
\hline $\begin{array}{l}\text { Duração da } \\
\text { residência }\end{array}$ & 3 & 2 & 2 & 2 & 2 & 0,075 \\
\hline $\begin{array}{l}\text { Foco em saúde } \\
\text { pública }\end{array}$ & 2 & 2 & 2 & 2 & 2 & 0,091 \\
\hline $\begin{array}{l}\text { Conselhos de } \\
\text { parentes }\end{array}$ & 2 & 2 & 2 & 2 & 3 & 0,091 \\
\hline Horas de trabalho & 3 & 3 & 3 & 4 & 4 & 0,092 \\
\hline $\begin{array}{l}\text { Flexibilidade da } \\
\text { especialidade }\end{array}$ & 4 & 4 & 3 & 4 & 3 & 0,115 \\
\hline $\begin{array}{l}\text { Interação com outros } \\
\text { médicos }\end{array}$ & 3 & 3 & 3 & 3 & 3 & 0,118 \\
\hline Qualidade de vida & 4 & 4 & 4 & 5 & 5 & 0,125 \\
\hline $\begin{array}{l}\text { Admiração a um } \\
\text { mentor }\end{array}$ & 2 & 2 & 2 & 2 & 3 & 0,125 \\
\hline $\begin{array}{l}\text { Tempo para se } \\
\text { dedicar à família }\end{array}$ & 4 & 4 & 4 & 4 & 4 & 0,178 \\
\hline Foco em emergência & 3 & 3 & 3 & 2 & 3 & 0,191 \\
\hline $\begin{array}{l}\text { Diversidade de } \\
\text { pacientes }\end{array}$ & 3 & 3 & 3 & 3 & 3 & 0,233 \\
\hline $\begin{array}{l}\text { Relação médico- } \\
\text { paciente }\end{array}$ & 4 & 4 & 4 & 4 & 4 & 0,294 \\
\hline $\begin{array}{l}\text { Compreender } \\
\text { paciente como um } \\
\text { todo }\end{array}$ & 4 & 3 & 4 & 3 & 4 & 0,360 \\
\hline $\begin{array}{l}\text { A pressão do dia } \\
\text { a dia }\end{array}$ & 3 & 3 & 3 & 3 & 3 & 0,396 \\
\hline
\end{tabular}

Os alunos que têm pais médicos dão maior relevância a conselho dos parentes; já os que não possuem pais médicos acreditam ser mais importante foco em saúde pública, faturamento precoce, interesse em relação a longo prazo com o paciente, diversidade de pacientes, tempo para lazer, compreender o paciente como um todo (biopsicossocial) e a relação médico-paciente. 
Na escolha da especialidade médica, os alunos foram divididos em três grupos. Os que ainda não haviam escolhido a especialidade, os que haviam escolhido uma especialidade geral (Cirurgia Geral, Clínica Médica, Pediatria, Ginecologia e Obstetrícia) e os que escolheram uma especialidade específica. Os fatores com maior significância estatística para o grupo que ainda não havia escolhido a especialidade foram compreender o paciente como um todo (biopsicossocial), pressão do dia a dia e foco em saúde pública. Para os que escolheram uma especialidade geral, os mais relevantes foram interesse em relação a longo prazo com o paciente, relação médico-paciente e prestígio. Para os que escolheram uma especialidade específica, foram horas de trabalho, qualidade de vida, tempo para o lazer, tempo para a família, recompensa financeira, departamento renomado, conselho de amigos e conselho de parentes.

\section{DISCUSSÃO}

A escolha de uma especialidade tende a dar resposta às características pessoais do estudante de Medicina, ou seja, os estudantes optam devido às percepções preexistentes baseadas na imagem que analisaram e interpretaram sobre determinada especialidade ao longo da sua vida pessoal e acadêmica ${ }^{39}$.

Neste estudo, observando que a maioria dos estudantes aponta a qualidade de vida e a recompensa financeira como dois dos principais motivos para a decisão da especialidade, é indiscutível que o estilo de vida se mostrou como prioridade. Isto pode ser devido a muitos determinantes que comprometem o estilo de vida de um médico, desde o início de sua formação com o ingresso na faculdade de Medicina, como durante o internato e a residência médica, até o dia a dia de um médico com maior tempo de formação.

Outra questão relevante é a oportunidade de emprego, que se mostrou como um dos fatores relevantes na escolha da especialidade. Historicamente, a profissão médica sempre foi vista com prestígio e respeito, principalmente pela relação existente entre o médico e o paciente. Porém, atualmente, os médicos estão sendo submetidos às regras impostas pelos sistemas de assistência à saúde (por exemplo, ritmo intenso de trabalho, instabilidade no emprego, jornadas prolongadas, salários não satisfatórios, pressão diante da obrigação de aliviar a dor dos pacientes e ter a morte como situação rotineira), o que acarreta desgaste físico e psicológico ${ }^{40}$. Essa preocupação é evidente em nosso estudo desde os primeiros anos da faculdade, mostrando-se como o primeiro fator de influência na escolha da especialidade entre os alunos do primeiro e segundo anos.

Desta forma, os resultados obtidos neste estudo refletem que a difícil decisão na escolha de uma especialidade é in- fluenciada por fatores que determinam principalmente o estilo de vida médico e a preocupação com a oportunidade de emprego.

\section{CONCLUSÃO}

Os resultados obtidos levam à análise de que, para este grupo de alunos do curso de Medicina, os fatores relacionados a qualidade de vida, retorno financeiro, relação médico-paciente e influências de terceiros foram os mais importantes na escolha das especialidades. O perfil financeiro do aluno não influenciou nessa escolha.

Trabalho realizado na disciplina de Técnica Cirúrgica do Departamento de Cirurgia da FCMSCSP.

O projeto de pesquisa foi aprovado pela comissão de ética: Projeto nº 368/10. Entrada no CEP: 06/10/10.

\section{REFERÊNCIAS}

1. Linn LS, Yager J, Cope D, Leake B. Health status, job satisfaction, job stress, and life satisfaction among academic and clinical faculty. JAMA 1985;254(19):2775-82.

2. Bergus GR, Randall CS, Winniford MD, Mueller CW, Johnson SR. Job satisfaction and workplace characteristics of primary and specialty care physicians at a bimodal medical school. Acad Med 2001;76(11):1148-52.

3. Linzer M, Konrad TR, Douglas J, McMurray JE, Pathman DE, Williams ES, et al. Managed care, time pressure, and physician job satisfaction: results from the physician worklife study. J Gen Intern Med 2000;15(7):441-50.

4. Maron BA, Fein S, Maron BJ, Hillel AT, El Baghdadi MM, Rodenhauser P. Ability of prospective assessment of personality profiles to predict the practice specialty of medical students. Proc (Bayl Univ Med Cent) 2007;20:22-6.

5. Conselho Federal de Medicina. Resolução CFM nº 1973/2011. Dispõe sobre a nova redação do Anexo II da Resolução CFM nº 1.845/08, que celebra o convênio de reconhecimento de especialidades médicas firmado entre o Conselho Federal de Medicina (CFM), a Associação Médica Brasileira (AMB) e a Comissão Nacional de Residência Médica (CNRM). Diário Oficial da União. Brasília, 1 ago. 2011; Seção 1, p. 144-7.

6. Markert RJ, Rodenhauser P, El-Baghdadi MM, Juskaite K, Hillel AT, Maron BA. Personality as a Prognostic Factor for Specialty Choice: A Prospective Study of 4 Medical School Classes. Medscape J Med 2008; 27;10(2):49.

7. Kiker BF, Zeh M. Relative income expectations, expected malpractice premium costs, and other determinants of physician specialty choice. J Health Soc Behav.1998;39:152-67. 
8. Burack JH, Irby DM, Carline JD, Ambrozy DM, Ellsbury KE, Stritter FT. A study of medical students' specialty-choice pathways: trying on possible selves. Acad Med.1997;72:534-41.

9. Borges NJ, Savickas ML. Personality and medical specialty choice: a literature review and integration. J Career Assess. 2002;10:362-80.

10. Mendes AS. Os estudantes de medicina: expectativas na escolha da especialidade. Lisboa; 2010. Mestrado [Dissertação] - Instituto Universitário de Lisboa.

11. McManus IC, Livingston G, Cornelius K. The attractions of medicine: the generic motivations of medical school applicants in relation to demography, personality and achievement. BMC Medical Education 2006; 6(11):1-15.

12. Murdoch MM, Kressin N, Fortier L, Giuffre PA, Oswald L. Evaluating the Psychometric Properties of a Scale to Measure Medical Students' Career related Value. Acad Med 2001; 76(2):157-65.

13. Zeldow PB, Devens M, Daugherty SR. Do person-oriented medical students choose person-oriented specialties? Do technology-oriented medical students avoid person-oriented specialties? Acad Med 1990;65(9 Suppl):S45-6.

14. Zeldow PB, Preston RC, Daugherty SR. The decision to enter a medical specialty: timing and stability. Med Educ 1992;26(4):327-32.

15. Zeldow PB, Daugherty SR. Personality profiles and specialty choices of students from two medical school classes. Acad Med 1991;66(5):283-7.

16. Kassebaum DG, Szenas PL. Factors influencing the specialty choices of 1993 medical school graduates. Acad Med 1994;69(2):163-70

17. Martini CJM, Veloski JJ, Barzansky B, Xu G, Fields SK. Medical school and student characteristics that influence choosing a generalist career. JAMA 1994;272(9):661-8.

18. Kassebaum DG, Szenas PL, Schuchert MK. Determinants of the generalist career intentions of 1995 graduating medical students. Acad Med 1996;71(2):198-209.

19. Gorenflo DW, Ruffin MT IV, Sheets KJ. A multivariate model for specialty preference by medical students. J Fam Pract 1994;39(6):570-6.

20. Vaidya NA, Sierles FS, Raida MD, Fakhoury FJ, Przybeck TR, Cloninger CR. Relationship between specialty choice and medical student temperament and character assessed with Cloninger Inventory. Teach Learn Med 2004;16(2):150-6.

21. Babbott D, Baldwin DC Jr, Killian CD, Weaver SO. Trends in evolution of specialty choice. Comparison of US medical school graduates in 1983 and 1987. JAMA 1989;261(16):2367-73. Erratum in JAMA1990;263(6):815.

22. Barshes NR, Vavra AK, Miller A, Brunicardi FC, Goss JA, Sweeney JF. General Surgery as a Career: A Contemporary Review of Factors Central to Medical Student Specialty Choice. J Am Coll Surg 2004;199(5):792-8.

23. Dorsey ER, Jarjoura D, Rutecki GW. Influence of Controllable Lifestyle on Recent Trends in Specialty Choice by US Medical Students. JAMA. 2003;290(9):1173-8.

24. Schwartz RW, Jarecky RK, Strodel WE, Haley JV, Young B, Griffen WO. Controllable lifestyle: a new factor in career choice by medical students. Acad Med.1989;64:606-9.

25. Pikoulis E, Avgerinos ED, Pedeli X, Karavokyros I, Bassios $\mathrm{N}$, Anagnostopoulou S. Medical students' perceptions on factors influencing a surgical career: The fate of general surgery in Greece. Surgery 2010;148(3):510-5.

26. Cruz EMTN. A escolha da especialidade em medicina. Campinas; 1976. Doutorado [Tese] - Faculdade de Ciências Médicas da Universidade Estadual de Campinas.

27. Figueiredo JF, Rodrigues ML, Troncon LEA, Cianflone AR. Influence of gender on specialty choices in a Brazilian medical school. Acad Med 1997;72(1):68-70.

28. Bellodi, PL. Clínica ou cirurgia - um estudo sobre razões da escolha da especialidade. São Paulo Med. J. 2004;122(3):81-6.

29. Coutts-van Dijk L, Bray JH, Moore S, Rogers J. Prospective study of how students' humanism and psychosocial beliefs relate to specialty matching. Acad Med 1997;72(12):1106-8.

30. Batenburg V, Smal JA, Lodder A, de Melker RA. Are professional attitudes related to gender and medical specialty? Med Educ 1999;33(7):489-92.

31. Vaglum P, Wiers-Jenssen J, Ekeberg O. Motivation for medical school: the relationship to gender and specialty preferences in a nationwide sample. Med Educ 1999;33(4):236-42.

32. Gorenflo DW, Ruffin MT 4th, Sheets KJ. A multivariate model for specialty preference by medical students. J Fam Pract 1994;39(6):570-6.

33. Fincher RM, Lewis LA, Jackson TW. Why students choose a primary care or nonprimary care career. The Specialty Choice Study Group. Am J Med 1994;97(5):410-7.

34. Osborn EH. Factors influencing students' choices of primary care or other specialties. Acad Med 1993;68(7):572-4.

35. Henry P, Leong FT, Robinson R. Choice of medical specialty: analysis of students' needs. Psychol ep 1992;71(1):215-24.

36. Kassler WJ, Wartman SA, Silliman RA. Why medical students choose primary care careers. Acad Med 1991;66(1):41-3. 
37. Rogers LQ, Fincher RM, Lewis LA. Factors influencing medical students to choose primary care or non-primary care specialties. Acad Med 1990;65(9 Suppl):S47-8.

38. Kassebaum DG, Szenas PL. Factors influencing the specialty choices of 1993 medical school graduates. Acad Med 1994;69(2):163-70.

39. Gaspar D. Medicina Geral e Familiar: Uma escolha gratificante. Acta Med Port 2006;19(2):133-40.

40. Oliveira GF, Barbosa GA, Souza LEC, Costa CLP, Araújo RCR, Gouveia VV. Satisfação com a vida entre profissionais da saúde: correlatos demográficos e laborais. Revista Bioética 2009;17(2):319-34.

\section{CONTRIBUIÇÃO DOS AUTORES}

Paulo Roberto Corsi: idealizador do estudo, coletou dados, contribuiu com a escrita do artigo científico. Erika Lopes Fernandes e Priscila Intelizano:realizaram revisão bibliográfica, realizaram análise estatística, escreveram a versão inicial do artigo científico, coletaram dados, tabelaram os dados, contribuíram com a escrita do artigo científico, revisaram a versão inicial do artigo, revisaram e escreveram a versão final do artigo, revisaram a análise estatística dos dados coletados. Manoel: orientou na metodologia científica, orientou na aplicação da estatística, revisou a analise estatística dos dados coletados.

\section{CONFLITO DE INTERESSES}

Declarou não haver.

\section{ENDEREÇO PARA CORRESPONDÊNCIA}

Paulo Roberto Corsi

Rua Antenor de Freitas, 140

Parque dos Príncipes - São Paulo

CEP.: 05396-010 - SP

E-mail: r.corsi@terra.com.br 